Check for updates

Cite this: RSC Adv., 2018, 8, 10017

\title{
The effect of alcohols as the third component on diffusion in mixtures of aromatics and ketones $\uparrow$
}

\author{
Tatjana Janzen, ${ }^{a}$ Yuri Gaponenko, ${ }^{\mathrm{b}}$ Aliaksandr Mialdun, (D) ${ }^{\mathrm{b}}$ \\ Gabriela Guevara-Carrion, ${ }^{a}$ Jadran Vrabec (D) *a and Valentina Shevtsova (D) *b
}

\begin{abstract}
With laboratory and numerical work, we demonstrate that one of the main diffusion coefficients and the smaller eigenvalue of the Fick diffusion matrix are invariant to the number of methylene groups of the alcohol in ternary mixtures composed of an aromatic (benzene), a ketone (acetone) and one of three different alcohols (methanol, ethanol or 2-propanol). A critical analysis of the relationship between the kinetic and thermodynamic contributions to the diffusion coefficients allows us to explain this intriguing behaviour of this class of mixture. These findings are reflected by the diffusive behaviour of the according binary subsystems. Our approach provides a promising systematic framework for future investigations into the important and challenging problem of transport diffusion in multicomponent liquids.
\end{abstract}

Received 1st February 2018

Accepted 27th February 2018

DOI: $10.1039 / \mathrm{c} 8 \mathrm{ra01007b}$

rsc.li/rsc-advances compounds were selected, i.e. an aromatic, a ketone and an alcohol. Throughout, the first two components were benzene (1) and acetone (2) and the third component was one of the alcohols, methanol, ethanol or 2-propanol. For each mixture, nine state points along a composition path with a constant content of benzene, $x_{1}=0.33 \mathrm{~mol} \mathrm{~mol}^{-1}$, were studied under ambient conditions (298.15 K and 0.1 MPa). Seven of the state points were ternary mixtures and two were binary subsystems. To obtain reliable results for the Fick diffusion coefficient matrix, two complementary approaches were used, i.e. experiments and predictive molecular simulations. This combination allows for a critical analysis and leads to a deeper understanding of the underlying phenomena. ${ }^{\mathbf{1 4 , 1 5}}$

The Taylor dispersion technique was utilized for the experiments. ${ }^{16,17}$ In this method, a small quantity of mixture with a slightly different composition is injected into a laminar stream. It disperses due to convection and diffusion while flowing through a capillary tube and the refractive index is measured at its end to sample the concentration distribution. We have used the same apparatus as in previous works. ${ }^{6,7}$ The Fick diffusion matrix is obtained by fitting working equations to the measured signal, i.e. the Taylor peak. The mathematical model of the Taylor dispersion technique was originally developed on the basis of Fick's law in the volume reference frame. In a ternary mixture, two molar fluxes $J_{i}^{v}$ relative to a volume averaged velocity are related to gradients of molar concentration $\nabla C_{i}$ with four diffusion coefficients $D_{i j}^{\mathrm{v}}$. Alternatively, fluxes expressed in the molar reference frame $J_{i}$ are relative to a molar averaged velocity and the mole fraction gradients $\nabla x_{i}$ act as a driving force

$$
\begin{aligned}
& J_{1} / \rho=-D_{11} \nabla x_{1}-D_{12} \nabla x_{2}, \\
& J_{2} / \rho=-D_{21} \nabla x_{1}-D_{22} \nabla x_{2},
\end{aligned}
$$


with molar density $\rho$. The fluxes of all three components are constrained by $\Sigma J_{i}=0$. The main diffusion coefficients $D_{11}$ and $D_{22}$ relate the flux of one component to its own mole fraction gradient and the cross diffusion coefficients $D_{12}$ and $D_{21}$ describe the coupling of the flux of one component with the gradient of the other. The third component does not appear in eqn (1) explicitly, but in general it affects all four diffusion coefficients. The transformation of experimental data from the volume to the molar reference frame $\left(D_{i j}^{\mathrm{v}}\right.$ to $\left.D_{i j}\right)$ could be done here on the basis of the pure component volumes (see the ESI $\dagger$ ).

Equilibrium molecular dynamics (MD) simulations were employed in this work, allowing for examination at the microscopic scale. The underlying molecular models were rigid, nonpolarizable force fields of united atom type, consisting of a varying number of Lennard-Jones, point charge, dipole and quadrupole sites (see the ESI $\dagger$ ). Note that the force field parameters were adjusted to pure fluid properties only so that all simulation results for the mixtures are strictly predictive. Diffusion coefficients were sampled with the Green-Kubo formalism, based on integrated correlation functions of net velocities of the contained species. ${ }^{\mathbf{1 1}, 15}$ Thereby, phenomenological coefficients $\Delta_{i j}$ were obtained, associating the diffusive fluxes with the chemical potential gradients $\nabla \mu_{i}$

$$
\begin{aligned}
& J_{1} / \rho=-\Delta_{11} \frac{x_{1}}{R T} \nabla \mu_{1}-\Delta_{12} \frac{x_{2}}{R T} \nabla \mu_{2}, \\
& J_{2} / \rho=-\Delta_{21} \frac{x_{1}}{R T} \nabla \mu_{1}-\Delta_{22} \frac{x_{2}}{R T} \nabla \mu_{2},
\end{aligned}
$$

with gas constant $R$ and temperature $T$. Fluxes $J_{i}$ correspond to the molar reference frame as in eqn (1).

The diffusion coefficients from experiment and simulation are related to different driving forces so that the chemical potential gradients have to be transformed to the mole fraction gradients for their comparison. ${ }^{18}$ This transformation is contained in the thermodynamic factor matrix $\boldsymbol{\Gamma}$

$$
\begin{aligned}
& {\left[\begin{array}{ll}
D_{11} & D_{12} \\
D_{21} & D_{22}
\end{array}\right]=\left[\begin{array}{ll}
\Delta_{11} & \Delta_{12} \\
\Delta_{21} & \Delta_{22}
\end{array}\right] \times\left[\begin{array}{ll}
\Gamma_{11} & \Gamma_{12} \\
\Gamma_{21} & \Gamma_{22}
\end{array}\right],} \\
& \Gamma_{i j}=\delta_{i j}+\left.x_{i} \frac{\partial \ln \gamma_{i}}{\partial x_{j}}\right|_{T, p, x_{k \neq j=1 \ldots N-1}}
\end{aligned}
$$

with the activity coefficient of species $i$ being $\gamma_{i}$, which expresses the non-ideality of a mixture with respect to the composition. This relationship shows that the Fick diffusion coefficients are actually the product of two contributions, a kinetic $\Delta_{i j}$ and a thermodynamic $\Gamma_{i j}$. The separate observation of these two contributions promotes understanding of the underlying physical phenomena. In the present study, the thermodynamic factor was calculated using the Wilson excess Gibbs energy $\left(\mathrm{g}^{\mathrm{E}}\right)$ model, using parameters fitted to experimental vapor-liquid equilibrium data of the binary subsystems (see the ESI $\dagger$ ). This combination of MD simulation results with a $\mathrm{g}^{\mathrm{E}}$ model was successfully used in previous work to predict Fick diffusion coefficients, including several binary subsystems of the ternary mixtures studied here. ${ }^{19}$

The four elements of the Fick diffusion coefficient matrix were determined for the three ternary mixtures, benzene + acetone + methanol/ethanol/2-propanol, for nine different compositions, each at ambient temperature and pressure.

Results for the first main element of the diffusion matrix $D_{11}$, which relates the flux of benzene to its own mole fraction gradient, are shown in Fig. 1(a). The experimental data agree quantitatively with the molecular simulation data. $D_{11}$ increases with the acetone content in the ternary mixture. Since mixtures with a constant mole fraction of benzene $\left(x_{1}=0.33 \mathrm{~mol} \mathrm{~mol}^{-1}\right)$ were studied throughout, the left edge of Fig. 1(a) corresponds to the binary limit of benzene + alcohol, while the right edge corresponds to that of benzene + acetone. Analysis of the ternary diffusive fluxes implies the following asymptotic behavior of the diffusion coefficients towards the binary limits: ${ }^{7}$ (i) at the infinite dilution limit, $x_{2} \rightarrow 0$, the ternary coefficient $D_{11}$ tends to the binary Fick diffusion coefficient of benzene + alcohol; (ii) at the other limit, $x_{3} \rightarrow 0, D_{11}-D_{12}=D_{22}-D_{21} \rightarrow D_{\text {bin }}$
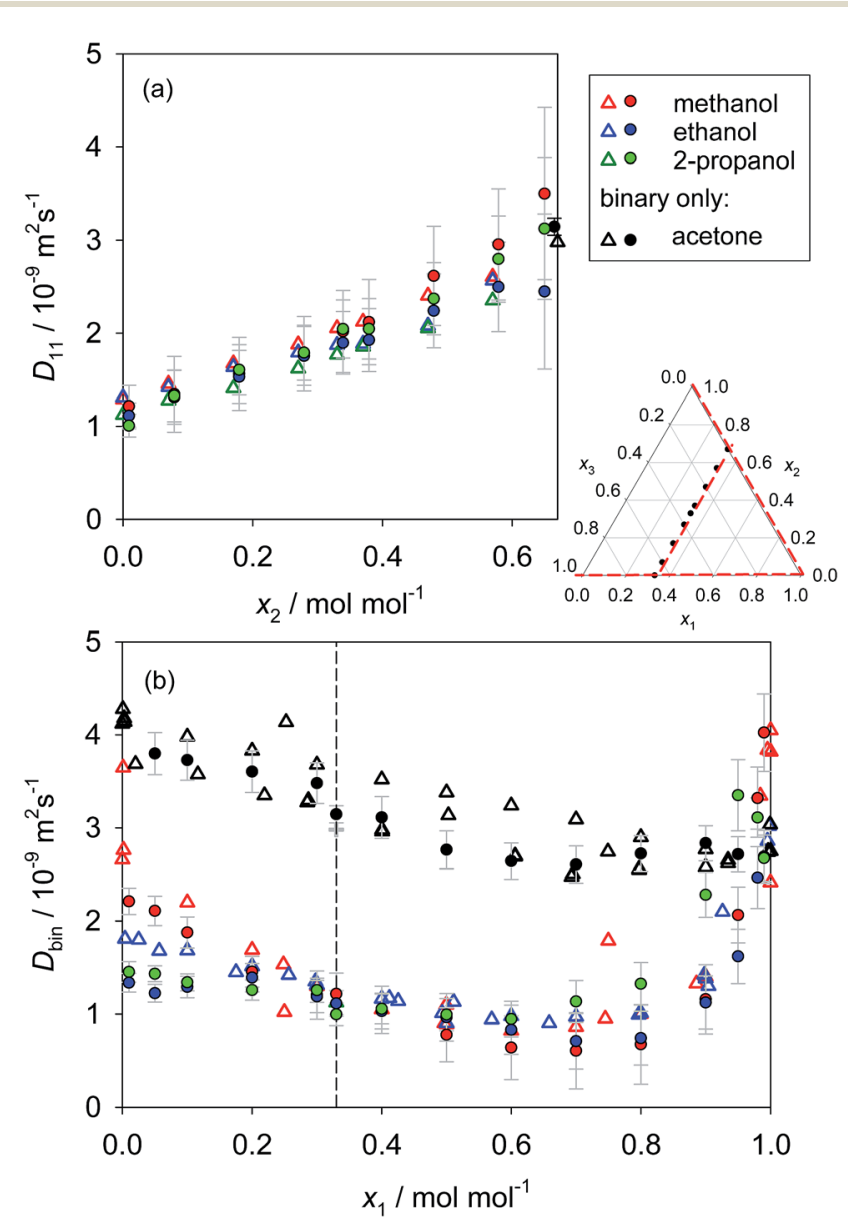

Fig. 1 Top: The main Fick diffusion coefficient (molar reference frame) of benzene $D_{11}$ in the three ternary mixtures benzene (1) + acetone (2) + alcohol (3) at a constant benzene mole fraction $x_{1}=0.33 \mathrm{~mol} \mathrm{~mol}^{-1}$ from experiment (triangles) and MD simulation combined with the Wilson $g^{E}$ model (circles). Both data sets were sampled at the same compositions, but are slightly shifted in the plot for visibility reasons. The symbols at the edges of this plot are the binary diffusion coefficients of benzene + alcohol $\left(x_{2} \rightarrow 0\right)$ and of benzene + acetone $\left(x_{3} \rightarrow\right.$ $0)$. Bottom: The binary Fick diffusion coefficient of the subsystems benzene + alcohol and benzene + acetone. Most of the binary experimental data were taken from the literature. ${ }^{20-27}$ 
(benzene + acetone) should hold. The present experimental and simulation results for $D_{11}$ are consistent with these asymptotic limits.

An inspection of Fig. 1(a) provides an unexpected finding: the main element $D_{11}$ is almost identical for all three mixtures along the examined composition path, i.e. it is independent of the contained type of alcohol. To explain this intriguing behavior of $D_{11}$, the properties of the pure components are considered first (see Table 1).

All five components are liquid under ambient conditions so that their self-diffusion coefficients are of the same order of magnitude. Molar masses $M$ and molar densities $\rho$, indicating the differences in mass and size of the molecules, give an introductory idea of their diffusion behavior. Benzene molecules are heavier and larger than acetone molecules, resulting in a self-diffusion coefficient $D^{0}$ that is only about half that of acetone. The three alcohols are characterized by increasing mass and size in the order: methanol, ethanol, 2-propanol. Although the methanol molecules are the smallest, due to hydrogen bonding interactions, their self-diffusion coefficient is similar to that of benzene. Molecules associated by hydrogen bonds propagate as an assembly, which significantly slows down their mobility. This is not only the case for methanol, but also for ethanol and 2-propanol. Because these molecules are larger, the bonded clusters are also larger and thus even slower. This hydrogen bonding behavior of the alcohol molecules causes micro-heterogeneity and cluster formation in mixtures with other fluids, ${ }^{28,29}$ which influences their kinetic and thermodynamic behavior.

Next, the binary subsystems of the ternary systems with different types of alcohol were examined. The Fick diffusion coefficient of the three binary benzene + alcohol mixtures and that of benzene + acetone is shown in Fig. 1(b). The benzene mole fraction, $x_{1}=0.33 \mathrm{~mol} \mathrm{~mol}^{-1}$, which was constant along the ternary composition path, is marked in the plot by a dashed vertical line. The binary Fick diffusion coefficient of all three benzene + alcohol mixtures has almost the same value in the concentration range around equimolar composition. However, at both infinite dilution limits $\left(x_{1} \rightarrow 0\right.$ and $\left.x_{1} \rightarrow 1\right)$, the benzene + methanol system has a higher Fick diffusion coefficient than the benzene + ethanol or benzene +2 -propanol systems. The open question is why the Fick diffusion coefficients are similar in a wide composition range. Although these binary data are available in the literature, to the best of our knowledge, they have never been discussed from this point of view.

Table 1 Pure component properties. The density and self-diffusion coefficient were obtained from MD simulation at $298.15 \mathrm{~K}$ and $0.1 \mathrm{MPa}$. The number in parentheses indicates the statistical uncertainty in the last digit

\begin{tabular}{lllll}
\hline & $M\left(\mathrm{~g} \mathrm{~mol}^{-1}\right)$ & $\rho\left(\mathrm{mol} \mathrm{l}^{-1}\right)$ & $\rho^{\mathrm{m}}\left(\mathrm{g} \mathrm{l}^{-1}\right)$ & $D^{0} 10^{-9}\left(\mathrm{~m}^{2} \mathrm{~s}^{-1}\right)$ \\
\hline Benzene & 78.11 & $11.147(2)$ & $870.6(1)$ & $2.226(4)$ \\
Acetone & 58.08 & $13.536(3)$ & $786.2(2)$ & $4.538(8)$ \\
Methanol & 32.04 & $24.541(6)$ & $786.3(2)$ & $2.449(6)$ \\
Ethanol & 46.07 & $17.132(4)$ & $789.3(2)$ & $0.974(3)$ \\
2-Propanol & 60.10 & $12.803(1)$ & $769.5(1)$ & $0.604(7)$
\end{tabular}

In a binary mixture there is only a single Fick diffusion coefficient and eqn (3) reduces to

$$
D=D \Gamma
$$

where $D$ is the Maxwell-Stefan (MS) diffusion coefficient. $D$ represents the kinetic contribution to the diffusion behavior, which was sampled here using MD simulations from net velocity correlation functions, while $T$ corresponds to the thermodynamic non-ideality, which was calculated using the Wilson $\mathrm{g}^{\mathrm{E}}$ model. Both contributions are separately shown in Fig. 2(a) and (c) for the three binary benzene + alcohol mixtures. The largest kinetic contribution, i.e. the MS diffusion coefficient, appears for benzene + methanol, followed by benzene + ethanol, which is also slightly larger than that of benzene +2 propanol (see Fig. 2(a)). The same order was observed for the self-diffusion coefficient of the pure alcohols, which also decreases from methanol over ethanol to 2-propanol. The nonideal composition dependence of the MS diffusion coefficient is a consequence of the hydrogen bonding behavior of the alcohols. The formation of clusters causes a correlated propagation of molecules. This leads to significant contributions of velocity correlations between unlike molecules, ${ }^{12,14,30}$ which are considered MS diffusion coefficient sampling (see the ESI $\dagger$ ). The thermodynamic factor exhibits the converse order: benzene mixed with methanol is the most non-ideal with the smallest thermodynamic factor, followed by ethanol and 2-propanol. Multiplying these two contributions leads to a similar Fick diffusion coefficient over a wide composition range of the three binary mixtures.

Building on this understanding, we further demonstrate that a similar interplay between kinetic and thermodynamic


Fig. 2 (a) The Maxwell-Stefan diffusion coefficient $\Theta$ of the three binary mixtures benzene + alcohol, (b) the phenomenological coefficient $\Delta_{11}$ of the ternary mixtures from MD simulation, (c) the thermodynamic factor $\Gamma$ of the three binary mixtures and (d) the thermodynamic factor $\Gamma_{11}$ of the ternary mixtures from the Wilson $\mathrm{g}^{\mathrm{E}}$ model. 
contributions is responsible for the independence of $D_{11}$ of the alcohol type for ternary mixtures of benzene and acetone with methanol, ethanol or 2-propanol. It follows from eqn (3) that $D_{11}=\Delta_{11} \Gamma_{11}+\Delta_{12} \Gamma_{21}$. Molecular simulation data show that the first term dominates the sum, while the second term is negligibly small. The kinetic $\Delta_{11}$ and thermodynamic $\Gamma_{11}$ contributions of the first term are depicted in Fig. 2(b) and (d). Indeed, as in the binary case, methanol exhibits the highest kinetic and the lowest thermodynamic contribution, providing that the product $\Delta_{11} \Gamma_{11}$ is the same for all considered types of alcohol. It can thus be concluded that the interplay between kinetics and thermodynamics leads to similar binary and ternary diffusion coefficients for mixtures of benzene and acetone with methanol, ethanol or 2-propanol. To examine the clustering behavior of the alcohols in the ternary mixtures, hydrogen bonding statistics were sampled using molecular simulations on the basis of geometric criteria ${ }^{31}$ (see Fig. 3). Most of the alcohol molecules are bonded to dimers and trimers within the ternary mixtures. The fractions of monomers, dimers, trimers and tetramers are almost identical for all three alcohols.

An important remaining question is whether the quantitative similarity of the binary and ternary diffusion coefficients can also relate to the second main Fick diffusion coefficient of the studied ternary mixtures. The diffusion coefficient $D_{22}$, characterizing the diffusive flux of acetone under its own mole fraction gradient, is shown in Fig. 4(a). The presence of benzene affects $D_{22}$, resulting in a less steep increase of that coefficient with higher acetone content. On average, $D_{22}$ is 1.5 to 2 times larger than $D_{11}$, which is in agreement with the twice as large self-diffusion coefficient of acetone compared to that of benzene. $D_{22}$ is fairly similar for ethanol and 2-propanol and noticeably higher for methanol. The binary diffusion coefficient of acetone + alcohol, shown in Fig. 3(b), resembles the behavior of $D_{22}$ in the ternary mixtures. As in the preceding discussion of $D_{11}$ and the corresponding binary subsystems, we decomposed the diffusion coefficient $D_{22}=\Delta_{21} \Gamma_{12}+\Delta_{22} \Gamma_{22}$ into its kinetic



Fig. 3 Hydrogen bonding statistics obtained from MD simulation in the three ternary mixtures benzene + acetone + alcohol, i.e. methanol (red), ethanol (blue) and 2-propanol (green), at a constant benzene mole fraction $x_{1}=0.33 \mathrm{~mol} \mathrm{~mol}^{-1}$.
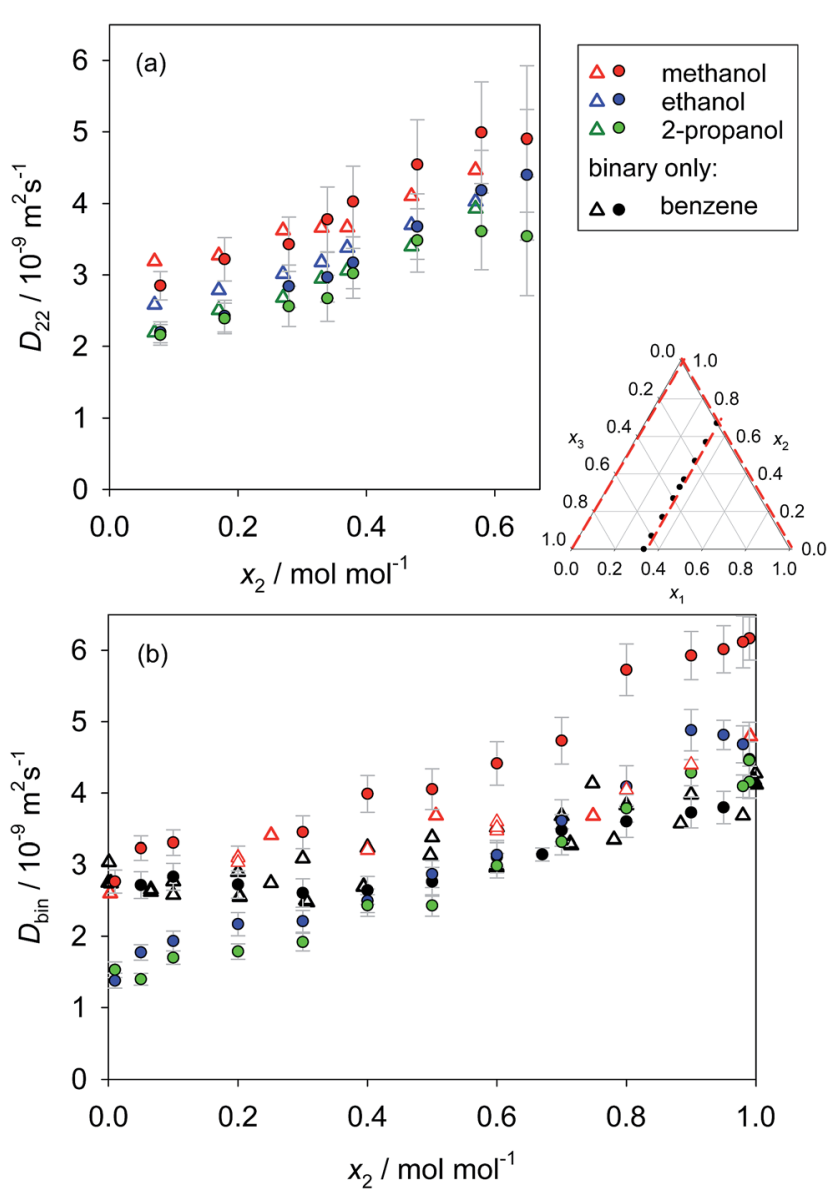

Fig. 4 Top: The main Fick diffusion coefficient (molar reference frame) of acetone $D_{22}$ in the three ternary mixtures benzene (1) + acetone (2) +alcohol (3) at a constant benzene mole fraction $x_{1}=$ $0.33 \mathrm{~mol} \mathrm{~mol}^{-1}$ from experiment (triangles) and MD simulation combined with the Wilson $\mathrm{g}^{\mathrm{E}}$ model (circles). Both data sets were sampled at the same compositions, but are slightly shifted in the plot for visibility reasons. Bottom: The binary Fick diffusion coefficient of the subsystems acetone + alcohol and acetone + benzene. Most of the binary experimental data were taken from the literature. ${ }^{20,24,25}$

and thermodynamic contributions. Molecular simulation data show that the cross term $\Delta_{21} \Gamma_{12}$ is again negligibly small. The kinetic contributions for the ternary $\Delta_{22}$ as well as for the binary $Ð$ (acetone + alcohol) mixtures are identical in the case of ethanol and 2-propanol, but much larger in the case of methanol. However, here the thermodynamic contributions for mixtures with methanol $\left(\Gamma_{22}\right.$ and $\left.\Gamma\right)$ cannot compensate for the large kinetic values. Separate analysis of kinetics and thermodynamics is a novel way for understanding diffusion.

We may thus draw the conclusion that for the liquid ternary mixtures benzene + acetone + alcohol, the qualitative behavior of the main coefficients $D_{11}$ and $D_{22}$ can directly be related to the binary subsystems, including the influence of contained alcohols on the composition dependent diffusion coefficients.

An important feature of ternary diffusion are the cross effects that cannot be related to binary behavior. As is often the case, the two cross coefficients of the studied ternary mixtures are significantly smaller than the main ones. The cross coefficient 



Fig. 5 Eigenvalues of the Fick diffusion coefficient matrix of the three ternary mixtures benzene (1) + acetone (2) + alcohol (3) at a constant benzene mole fraction $x_{1}=0.33 \mathrm{~mol} \mathrm{~mol}^{-1}$ from experiment (triangles) and MD simulation combined with the Wilson $\mathrm{g}^{\mathrm{E}}$ model (circles).

of benzene $D_{12}$, relating the flux of benzene to the mole fraction gradient of acetone, has mostly small negative values for all three ternary mixtures, except for small alcohol concentrations in the mixture with methanol, where it is positive. The second cross coefficient of acetone $D_{21}$ must be zero at the limit $x_{2} \rightarrow 0$, which is confirmed by the trend of the data. At the other limit $x_{3}$ $\rightarrow 0$, the coefficients are positive and increasing towards the limit of diluted alcohol, with the highest values in the mixture with methanol and the lowest for 2-propanol.

In contrast to the individual elements of the Fick diffusion coefficient matrix, the eigenvalues of the matrix do not depend on the reference frame or on the order of components. Furthermore, a constraint imposed by the second law of thermodynamics is that the eigenvalues of the Fick diffusion coefficient matrix must be real and positive for a thermodynamically stable mixture. The eigenvalues of the diffusion matrix obtained by experiment and simulation fulfil these specifications. They show the same variation with composition and dependence on the type of alcohol, which was already observed for the main elements of the diffusion matrix (see Fig. 5). The larger eigenvalue $D_{1}$ increases with acetone content and shows higher values in the ternary mixture with methanol, while it is slightly lower for ethanol and 2-propanol. This correlates with the behavior of $D_{22}$. The smaller eigenvalue $D_{2}$, like the main coefficient $D_{11}$, is independent of the type of alcohol for the three studied ternary mixtures.

Fick diffusion coefficients of three different ternary mixtures, i.e. benzene + acetone + methanol/ethanol/2propanol, were analyzed. Two complementary approaches were utilized to obtain reliable data, experiments and molecular simulation. We identified an important feature of this class of mixture (an aromatic, a ketone and an alcohol): namely that one of the main diffusion coefficients $D_{11}$, where $D_{11}<D_{22}$, and the smaller eigenvalue $D_{2}$ are independent of the alcohol type along the studied composition path. This insight was reflected in another finding that the Fick diffusion coefficient of the binary benzene + alcohol subsystems also does not depend on the alcohol type. The underlying mechanism of this unusual behavior was explained by separately considering the kinetic and thermodynamic contributions to the diffusion coefficients. The results presented here provide a promising framework for future systematic investigations into the important and challenging problem of diffusion in multicomponent liquid mixtures. In order to provide a more substantial understanding of phenomena occurring in multicomponent mixtures, the present study can be continued and extended by replacing one main component of the ternary mixture, e.g. benzene, with another aromatic substance, e.g. toluene.

\section{Conflicts of interest}

There are no conflicts to declare.

\section{Acknowledgements}

The authors acknowledge financial support from Deutsche Forschungsgemeinschaft (grant VR 6/11-1) and the Belgian Federal Science Policy Office (PRODEX program). The simulations were carried out on the national supercomputer Hazel Hen at the High Performance Computing Center Stuttgart within the project MMHBF2.

\section{Notes and references}

1 F. Ingremeau, M. Dolega, J. Gallagher, I. Wang, G. Cappello and A. Delon, Soft Matter, 2017, 13, 4210-4213.

2 C. Forrey, D. M. Saylor, J. S. Silverstein, J. F. Douglas, E. M. Davis and Y. A. Elabd, Soft Matter, 2014, 10, 7480-7494.

3 S. Ye, Q. Tang, J. Yang, K. Zhang and J. Zhao, Soft Matter, 2016, 12, 9520-9526.

$4 \mathrm{~J}$. Wambui Mutoru and A. Firoozabadi, J. Chem. Thermodyn., 2011, 43, 1192-1203.

5 V. K. Vanag and I. R. Epstein, Phys. Chem. Chem. Phys., 2009, 11, 897-912.

6 J. C. Legros, Y. Gaponenko, A. Mialdun, T. Triller, A. Hammon, C. Bauer, W. Kohler and V. Shevtsova, Phys. Chem. Chem. Phys., 2015, 17, 27713-27725.

7 V. Sechenyh, J. C. Legros, A. Mialdun, J. M. Ortiz de Zárate and V. Shevtsova, J. Phys. Chem. B, 2016, 120, 535-548. 
8 A. Heller, C. Giraudet, Z. A. Makrodimitri, M. S. H. Fleys, J. Chen, G. P. van der Laan, I. G. Economou, M. H. Rausch and A. P. Fröba, J. Phys. Chem. B, 2016, 120, 10808-10823.

9 C. Peters, L. Wolff, S. Haase, J. Thien, T. Brands, H.-J. Koß and A. Bardow, Lab Chip, 2017, 17, 2768-2776.

$10 \mathrm{X}$. Zhang, A. Poniewierski, A. Jelińska, A. Zagożdżon, A. Wisniewska, S. Hou and R. Hołyst, Soft Matter, 2016, 12, 8186-8194.

11 R. Krishna and J. M. van Baten, Ind. Eng. Chem. Res., 2005, 44, 6939-6947.

12 X. Liu, T. J. Vlugt and A. Bardow, Ind. Eng. Chem. Res., 2011, 50, 10350-10358.

13 T. Allie-Ebrahim, Q. Zhu, P. Bräuer, G. D. Moggridge and C. D’Agostino, Phys. Chem. Chem. Phys., 2017, 19, 1607116077.

14 T. Janzen, S. Zhang, A. Mialdun, G. Guevara-Carrion, J. Vrabec, M. He and V. Shevtsova, Phys. Chem. Chem. Phys., 2017, 19, 31856-31873.

15 G. Guevara-Carrion, Y. Gaponenko, T. Janzen, J. Vrabec and V. Shevtsova, J. Phys. Chem. B, 2016, 120, 12193-12210.

16 W. A. Wakeham, Faraday Symp. Chem. Soc., 1980, 15, 145154.

17 W. E. Price, J. Chem. Soc., Faraday Trans. 1, 1988, 84, 24312439.

18 R. Taylor and H. A. Kooijman, Chem. Eng. Commun., 1991, 102, 87-106.
19 G. Guevara-Carrion, T. Janzen, Y. M. Munoz-Munoz and J. Vrabec, J. Chem. Phys., 2016, 144, 124501.

20 A. Alimadadian and C. P. Colver, Can. J. Chem. Eng., 1976, 54, 208-213.

21 C. C. Caldwell and A. L. Baab, J. Phys. Chem., 1955, 59, 11131114.

22 D. K. Anderson, J. R. Hall and A. L. Babb, J. Phys. Chem., 1958, 62, 404-408.

23 R. R. Irani and A. W. Adamson, J. Phys. Chem., 1960, 64, 199204.

24 H. T. Cullinan and H. L. Toor, J. Phys. Chem., 1965, 69, 39413949.

25 D. W. McCall and D. C. Douglass, J. Phys. Chem., 1967, 71, 987-997.

26 S. S. Rao and C. O. Bennett, AIChE J., 1971, 17, 75-81.

27 M. Zhou, X. Yuan, Y. Zhang and K. T. Yu, Ind. Eng. Chem. Res., 2013, 52, 10845-10852.

28 A. Perera, L. Zoranić, F. Sokolić and R. Mazighi, J. Mol. Liq., 2011, 159, 52-59.

29 M. Požar, J.-B. Seguier, J. Guerche, R. Mazighi, L. Zoranić, M. Mijaković, B. Kežić-Lovrinčević, F. Sokolić and A. Perera, Phys. Chem. Chem. Phys., 2015, 17, 9885-9898.

30 H. Weingärtner, Ber. Bunsen-Ges., 1990, 94, 358-364.

31 M. Haughney, M. Ferrario and I. R. McDonald, J. Phys. Chem., 1987, 91, 4934-4940. 